\title{
NEW CHARACTERIZATIONS FOR \\ HANKEL TRANSFORMABLE SPACES OF ZEMANIAN
}

\author{
J. J. BETANCOR \\ Departamento de Analisis Matematico \\ Facultad de Matematıcas \\ Universidad de La Laguna \\ 38271 La Laguna, Tenerife \\ Islas Canarias, SPAIN
}

(Received April 11, 1994 and in revised form February 25,1995)

ABSTRACT. In this paper we obtain new characterizations of the Zemanian spaces $H_{\mu}$ and $H_{\mu}$

KEY WORDS AND PHRASES. Hankel transform, distribution, Zemanian spaces AMS SUBJECT CLASSIFICATION CODE. 46F 12

A $\mathrm{H}$ Zemanian [7, Ch 5] introduced the space $H_{\mu}(\mu \in \mathbb{R})$ of functions as follows a complex valued smooth function $\phi(x), x \in I=(0, \infty)$, is in $H_{\mu}$ if, and only if, the quantity

$$
\gamma_{n, h}^{\prime \prime}(\phi)=\sup _{x=1}\left|x^{n}\left(x^{-1} D\right)^{h}\left(x^{-\mu-1 / 2} \phi(x)\right)\right|<\infty
$$

is finite, for every $n, k \in \mathbb{N}$. This space endowed the topology generated by $\left\{\gamma_{n, k}^{\mu}\right\}_{n, k \in \mathbb{N}}$ is a Fréchet space In the sequel we will refer to the above topology as the usual topology of $H_{\mu} \quad$ Zemanian introduced the space $H_{\mu}$ to extend the Hankel integral transformation defined by

$$
\left(h_{\mu} \phi\right)(x)=\int_{0}^{\infty}(x t)^{1 / 2} J_{\mu}(x t) \phi(t) d t
$$

where $J_{\mu}$ denotes the Bessel function of the first kind and order $\mu$, to generalized functions. He proved that $h_{\mu}$ is an automorphism of $H_{\mu}$ provided that $\mu \geq \frac{1}{2}$. The generalized Hankel transform $h$ fof $f \in H_{\mu}^{\prime}$, the dual space of $H_{\mu}$, is defined as the transposed of $H_{\mu}$ through

$$
\left\langle h_{\mu}^{\prime} f, \phi\right\rangle=\left\{, h_{\mu} \phi\right\rangle \quad \text { for } \quad \phi \in H_{\mu} .
$$

Thus if $\mu \geq \frac{1}{2} \quad h$ is an automorphism of $\not \mu$ when this space is equipped with the weak* topology or with the strong topology.

In [2] J. J. Betancor and I. Marrero have studied the main topological properties of the spaces $H_{\mu}$ and $H_{\mu}^{\prime}$. Amongst other results, it is established (Theorem 3.3) that the space $H_{\mu}, \mu \geq \frac{1}{2}$, is constituted by all those complex valued smooth functions $\phi(x), x \in I$, such that

$$
\tau_{n, k}^{\mu}(\phi)=\sup _{x \in I}\left|x^{n} N_{\mu+k-1} \ldots N_{\mu} \phi(x)\right|<\infty
$$

for every $n, k \in \mathbb{N}$. Moreover, the system of seminorms $\left\{\tau_{n, k}^{\mu}\right\}_{n, k \in \mathbb{N}}$ generates of $H_{\mu}$ its usual topology Moreover in [4] they gave new descriptions for the usual topology of $H_{\mu}$ through $L_{22}$-norms

A. H. Zemanian [7, p. 134] defined the space $O$ formed by all those complex valued smooth functions $v(x), x \in I$, satisfying that for every $k \in \mathbb{N}$ there exists $n_{k} \in \mathbb{N}$ such that $\left(1+x^{2}\right)^{n_{k}}\left(x^{-1} D\right)^{k} v(x)$ is a bounded function on $I$. He proved that $O$ is a space of multiplier of $H_{\mu}$ Recently J. J. Betancor and I. Marrero [2, Theorems 2.3 and 4.9] have characterized $O$ as the space of multipliers of $H_{\mu}$ and $H_{\mu}^{\prime}$.

In this paper we characterize the smooth complex valued functions in $H_{\mu}, \mu \geq \frac{1}{2}$, as the ones satisfying

$$
Z_{n}(\phi)=\sup _{x \in I}\left|x^{n} \phi(x)\right|<\infty
$$


and

$$
y_{n}^{\mu}(\phi)=\sup _{x \in I}\left|N_{\mu+n-1} \ldots N_{\mu} \phi(x)\right|<\infty
$$

for every $n \in N$. Moreover we prove that the usual topology of $H_{\mu}$ can be defined by the family of seminorms $\left\{Z_{n}, y_{n}^{\mu}\right\}_{n \in \mathbf{N}}$ and a new characterization for the elements of $H_{\mu}^{\prime}$ is obtained. In the sequel we will assume that $\mu \geq-\frac{1}{2}$.

PROPOSITION 1. A complex valued smooth function $\phi(x), x \in I$, is in $H_{\mu}$ if, and only if, $\phi$ satisfies (1) and (2) for every $n \in \mathbb{N}$.

PROOF. It is clear that if $\phi \in H_{\mu}$ then $\phi$ satisfies (1) and (2) for every $n \in \mathbb{N}$.

Let now $\phi$ be a complex valued smooth function defined on $I$. To see that (1) and (2) $(n \in \mathbb{N})$ are sufficient conditions for $\phi$ belongs to $H_{\mu}$ we proceed by induction. Suppose, as induction hypothesis, that

$\sup _{\substack{x \in I \\ \text { for certain } \ell}} \mid x^{m} N_{\mu+}, \ell \geq 1$.

$$
\sup _{x \in I}\left|x^{m} N_{\mu+n-1} \ldots N_{\mu} \phi(x)\right|<\infty, \quad m \in \mathbb{N} \quad \text { and } \quad n \in \mathbb{N}, 0 \leq n<\ell
$$

By using partial integration we can obtain

$$
\begin{aligned}
\left\|x^{m} N_{\mu+\ell-1} \ldots N_{\mu} \phi(x)\right\|_{2}^{2} & =\int_{0}^{\infty}\left|x^{m} N_{\mu+\ell-1} \ldots N_{\mu} \phi(x)\right|^{2} d x \\
& =\int_{0}^{\infty} x^{2 m} N_{\mu+\ell-1} \ldots N_{\mu}(\phi(x)) N_{\mu+\ell-1} \ldots N_{\mu}(\bar{\phi}(x)) d x \\
& =\int_{0}^{\infty}\left(D x^{-1}\right)^{\ell}\left(x^{2 m+\mu+\ell+1 / 2} N_{\mu+\ell-1} \ldots N_{\mu}(\phi(x))\right) x^{-\mu-1 / 2} \bar{\phi}(x) d x
\end{aligned}
$$

for every $m \in \mathbb{N}, \quad \ell<2 m+2$, because

$$
\left[\left(D x^{-1}\right)^{2}\left(x^{2 m+\mu+\ell+1 / 2} N_{\mu+\ell-1} \ldots N_{\mu}(\phi(x))\right)\left(x^{-1} D\right)^{\ell-\imath-1}\left(x^{-\mu-1 / 2} \bar{\phi}(x)\right)\right]_{0}^{\infty}=0
$$

for each $i, m \in \mathbb{N}, 0 \leq i<\ell<2 m+2$. In effect, if $m, i \in \mathbb{N}, \quad 0 \leq i<\ell<2 m+2$ then Leibniz's rule leads to

$$
\begin{aligned}
\left(D x^{-1}\right)^{i} & \left(x^{2 m+\mu+\ell+1 / 2} N_{\mu+\ell-1} \ldots N_{\mu}(\phi(x))\right)\left(x^{-1} D\right)^{\ell-\imath-1}\left(x^{-\mu-1 / 2} \bar{\phi}(x)\right) \\
& =\sum_{j=0}^{\imath} a_{j} x^{2 m+2 \ell+2 \mu+1-2 j}\left(x^{-1} D\right)^{\ell+\imath-j}\left(x^{-\mu-1 / 2} \phi(x)\right)\left(x^{-1} D\right)^{\ell-i-1}\left(x^{-\mu-1 / 2} \bar{\phi}(x)\right) \\
& =\sum_{j=0}^{i} a_{j} x^{2 m+1-j} N_{\mu+\ell+i-j-1} \ldots N_{\mu}(\phi(x)) N_{\mu+\ell-i-2} \ldots N_{\mu}(\phi(x))
\end{aligned}
$$

where $a_{j}, \quad j \in \mathbb{N}, 0 \leq j \leq i$, are suitable real numbers, and by virtue of induction hypothesis (3) follows.

Most straightforward manipulations allow us to write

$$
\left(D x^{-1}\right)^{\ell}\left(x^{2 m+\mu+\ell+1 / 2} N_{\mu+\ell-1} \ldots N_{\mu}(\phi(x))\right) x^{-\mu-1 / 2} \bar{\phi}(x)=\sum_{j=0}^{\ell} a_{j} x^{2 m-j} \bar{\phi}(x) N_{\mu+2 \ell-j-1} \ldots N_{\mu} \phi(x)
$$

with $m \in \mathbb{N}$ and $a_{j} \in \mathbb{R}, \quad j \in \mathbb{N}, \quad 0 \leq j \leq \ell$.

Hence we can establish

$$
\begin{aligned}
& \left\|x^{m} N_{\mu+\ell-1} \ldots N_{\mu} \phi(x)\right\|_{2}^{2} \leq C_{1} \sum_{j=0}^{\ell} \int_{0}^{\infty}\left|x^{2 m-\jmath} \bar{\phi}(x)\right|\left|N_{\mu+2 \ell-j-1} \ldots N_{\mu} \phi(x)\right| d x \\
& \leq C_{2} \sum_{j=0}^{\ell} \sup _{x \in I}\left|\left(1+x^{2}\right) x^{2 m-j} \phi(x)\right| \sup _{x \in I}\left|N_{\mu+2 \ell-j-1} \ldots N_{\mu} \phi(x)\right|<\infty,
\end{aligned}
$$


provided that $m \in \mathbb{N}, 2 m \geq \ell$. Here $C_{\imath}, \quad i=1,2$, denotes suitable positive constants.

Assume now that $m \in \mathbb{N}, 2 m<\ell$. We have

$$
\begin{aligned}
\left\|x^{m} N_{\mu+\ell-1} \ldots N_{\mu} \phi(x)\right\|_{2}^{2}= & \left(\int_{0}^{1}+\int_{1}^{\infty}\right)\left|x^{m} N_{\mu+\ell-1} \ldots N_{\mu} \phi(x)\right|^{2} d x \\
& \leq \int_{0}^{1}\left|N_{\mu+\ell-1} \ldots N_{\mu} \phi(x)\right|^{2} d x+\int_{0}^{\infty}\left|x^{\ell} N_{\mu+\ell-1} \ldots N_{\mu} \phi(x)\right|^{2} d x .
\end{aligned}
$$

Therefore, by invoking (4) and the induction hypothesis we infer that

$$
\left\|x^{m} N_{\mu+\ell-1} \ldots N_{\mu} \phi(x)\right\|_{2}<\infty, \quad \text { when } \quad m \in \mathbb{N}, 2 m \leq \ell .
$$

Thus it is concluded that $\left\|x^{m} N_{\mu+\ell-1} \ldots N_{\mu} \phi(x)\right\|_{2}<\infty, \quad m \in \mathbb{N}$.

Also, for every $m \in \mathbb{N}, \quad m \geq 1$, and $x \in I$,

$$
\begin{aligned}
& \left(x^{m} N_{\mu+\ell-1} \ldots N_{\mu} \phi(x)\right)^{2}=\int_{0}^{x} D_{t}\left(t^{m} N_{\mu+\ell-1} \ldots N_{\mu} \phi(t)\right)^{2} d t \\
& \quad=\int_{0}^{x} 2 t^{m} N_{\mu+\ell-1} \ldots N_{\mu}(\phi(t))\left(\left[m+\mu+\frac{1}{2}+\ell\right] t^{m-1} N_{\mu+\ell-1} \ldots N_{\mu}(\phi(t))+t^{m} N_{\left.\mu+\ell \ldots N_{\mu}(\phi(t))\right) d t .}\right.
\end{aligned}
$$

Hence if $m \in \mathbb{N}, m \geq 1$, and $x \in I$ by using Holder's inequality we can find $C \geq 0$ such that

$$
\begin{aligned}
& \left|x^{m} N_{\mu+\ell-1} \ldots N_{\mu} \phi(x)\right|^{2} \leq C\left(\left\|x^{m} N_{\mu+\ell-1} \ldots N_{\mu} \phi(x)\right\|_{2}\left\|x^{m-1} N_{\mu+\ell-1} \ldots N_{\mu} \phi(x)\right\|_{2}\right. \\
& \left.+\sup _{x \in I}\left|N_{\mu+\ell} \ldots N_{\mu} \phi(x)\right|\left[\left\|x^{m} N_{\mu+\ell-1} \ldots N_{\mu} \phi(x)\right\|_{2}+\left\|x^{m+1} N_{\mu+\ell-1} \ldots N_{\mu} \phi(x)\right\|_{2}\right]\right)
\end{aligned}
$$

and then $\sup _{x \in I}\left|x^{m} N_{\mu+\ell-1} \ldots N_{\mu} \phi(x)\right|<\infty, m \in \mathbb{N}$.

Thus the proof is finished.

The last proposition allows us to define the usual topology of $H_{\mu}$ through a family of seminorms simpler than $\left\{\gamma_{m, k}^{\mu}\right\}_{m, k \in \mathbf{N}}$.

PROPOSITION 2. The usual topology of $H_{\mu}$ is defined by the system of seminorms $\left\{Z_{n}, y_{n}^{\mu}\right\}_{n \in \mathbf{N}}$.

PROOF. It is clear that the topology generated by $\left\{\gamma_{m, k}^{\mu}\right\}_{m, k \in N}$ is finer than the one defined by $\left\{Z_{n}, y_{n}^{\mu}\right\}_{n \in \mathbf{N}}$ on $H_{\mu}$. Moreover by proceeding in a way similar to A. H. Zemanian [7, Lemma 5.2-2] we can prove that $H_{\mu}$ endowed with the topology generated by $\left\{Z_{n}, y_{n}^{\mu}\right\}_{n \in \mathbf{N}}$ is a Fréchet space. Hence the desired result is an immediate consequence of the Open Mapping Theorem [6, Corollary 2.12].

We now prove a new characterization for the elements of $H_{\mu}^{\prime}$ the dual space of $H_{\mu}$. The procedure employed is analogous to the one used by the author [1] and by J. J. Betancor and I. Marrero [2].

PROPOSITION 3. Let $f$ be a linear functional defined on $H_{\mu}$. Then $f$ is in $H_{\mu}^{\prime}$ if, and only if, there exist $r \in \mathbb{N}$ and $f_{k}, g_{k} \in L_{\infty}(0, \infty)$ (the space of essentially bounded functions on $(0, \infty)$ ), $k \in \mathbb{N}, 0 \leq k \leq r$, such that

$$
f=\sum_{k=0}^{r} h_{\mu}^{\prime}\left(x^{k} f_{k}+x^{-\mu+1 / 2}\left(x^{-1} D\right)^{k} x^{k+\mu-1 / 2} g_{k}\right) .
$$

PROOF. Let $f \in H_{\mu}^{\prime}$. By virtue of a well-known result ([7, Theorem 1.8-1]) there exist $r \in \mathbb{N}$ and $C>0$ such that

$$
|\langle f, \phi\rangle| \leq C \max _{0 \leq \mathbf{k} \leq \mathrm{r}}\left\{Z_{k}(\phi), y_{k}^{\mu}(\phi)\right\}, \quad \phi \in H_{\mu}
$$

According to [7, Lemma 5.4-1(2), (3) and Theorem 5.4-1] and since $z^{1 / 2} J_{\mu}(z)$ is a bounded function on $I$ for every $k \in \mathbb{N}$ one has 


$$
\sup _{x \in I}\left|x^{k} \phi(x)\right|=\sup _{x \in I}\left|x^{k} h_{\mu}\left(h_{\mu} \phi\right)(x)\right| \leq C \int_{0}^{\infty}\left|N_{\mu+k-1} \ldots N_{\mu}\left(h_{\mu} \phi\right)(t)\right| d t
$$

and

$$
\sup _{x \in I}\left|N_{\mu+k-1} \ldots N_{\mu} \phi(x)\right|=\sup _{x \in I}\left|N_{\mu+k-1} \ldots N_{\mu} h_{\mu}\left(h_{\mu} \phi\right)(x)\right| \leq C \int_{0}^{\infty}\left|t^{k}\left(h_{\mu} \phi\right)(t)\right| d t
$$

for a suitable $C>0$.

The linear mapping

$$
\begin{aligned}
j: H_{\mu} & \rightarrow J H_{\mu} \subset L_{1}(0, \infty)^{2 r+2} \\
\phi & \rightarrow\left(x^{k} h_{\mu} \phi, N_{\mu+k-1} \ldots N_{\mu} h_{\mu} \phi\right)_{k=0}^{r}
\end{aligned}
$$

is one to one because $h_{\mu}$ is an automorphism of $H_{\mu}$ ([7, Theorem 5.4-1]). Here $L_{1}(0, \infty)$ denotes the usual Lebesgue space of order 1 .

On the other hand, the inequalities (6), (7) and (8) imply that the linear mapping

$$
\begin{aligned}
L: J H_{\mu} \subset L_{1}(0, \infty)^{2 r+2} & \rightarrow \mathbb{C} \\
\left(x^{k} h_{\mu} \phi, N_{\mu+k-1} \ldots N_{\mu} h_{\mu} \phi\right)_{k=0}^{r} & \rightarrow\langle f, \phi\rangle
\end{aligned}
$$

is continuous when $J H_{\mu}$ is endowed with the topology induced by $L_{1}(0, \infty)^{2 r+2}$. Hence, by invoking the Hahn-Banach Theorem $L$ can be extended to $L_{1}(0, \infty)^{2 r+2}$ as a member of $\left(L_{1}(0, \infty)^{2 r+2}\right)^{\prime}$, the dual space of $L_{1}(0, \infty)^{2 r+2}$. Since, as it is well known, $L_{1}(0, \infty)^{\prime}=L_{\infty}(0, \infty)$ there exist $f_{k}$, $g_{k} \in L_{\infty}(0, \infty), k \in \mathbb{N}, 0 \leq k \leq r$, such that

$$
\langle f, \phi\rangle=\sum_{k=0}^{r}\left(\left\langle f_{k}, x^{k} h_{\mu} \phi\right\rangle+\left\langle g_{k}, x^{k+\mu+1 / 2}\left(x^{-1} D\right)^{k}\left(x^{-\mu-1 / 2} \phi\right)\right\rangle\right), \quad \phi \in H_{\mu} .
$$

Therefore

$$
f=\sum_{k=0}^{r} h_{\mu}^{\prime}\left(x^{k} f_{k}+(-1)^{k} x^{-\mu+1 / 2}\left(x^{-1} D\right)^{k} x^{k+\mu-1 / 2} g_{k}\right) .
$$

Thus the proof of necessity if finished.

Conversely, if $f$ is a linear functional defined on $H_{\mu}$ by (5) for certain $r \in \mathbb{N}$ and $f_{k}$, $g_{k} \in L_{\infty}(0, \infty), k \in \mathbb{N}, 0 \leq k \leq r$, then

$$
|\langle f, \phi\rangle| \leq C \sum_{k=0}^{r}\left(\left\|f_{k}\right\|_{\infty} \sup _{x \in I}\left|\left(1+x^{2}\right) x^{k}\left(h_{\mu} \phi\right)(x)\right|+\left\|g_{k}\right\|_{\infty} \sup _{x \in I}\left|\left(1+x^{2}\right) N_{\mu+k-1} \ldots N_{\mu}\left(h_{\mu} \phi\right)(x)\right|\right)
$$

for $\phi \in H_{\mu}$, where $\|\cdot\|_{\infty}$ denotes the usual norm in $L_{\infty}(0, \infty)$. Hence, according to [7, Theorem 5.4-1] and [2, Theorem 3.3], $f$ is in $H_{\mu}^{\prime}$.

\section{REFERENCES}

[1] BETANCOR, J.J., Characterization of Hankel transformable generalized functions, Internat. J. Math. \& Math. Sci., 14 (2) (1991), 269-274.

[2] BETANCOR, J.J. and MARRERO, I., Some linear topological properties of the Zemanian space $H_{\mu}$, Bull. de la Soc. Roy. des Sci. de Liege, 61 (3-4) (1992), 299-314.

[3] BETANCOR, J.J. and MARRERO, I., Multipliers of Hankel transformable generalized functions, Comment. Math. Univ. Carolinae, 33 (3) (1992), 389-401.

[4] BETANCOR, J.J. and MARRERO, I., A Hilbert space approach to Hankel transformable distributions, Applicable Analysis, 52 (1994), 103-124.

[5] CHUNG, J., CHUNG, S-Y. and KIM, D., Une caractérisation de l'espace S de Schwartz, C. $R$. Acad. Sci. Paris, 316 Serie I (1993), 23-25.

[6] RUDIN, W., Functional Analysis (Second Edition), McGraw-Hill, Inc., New York, 1991.

[7] ZEMANIAN, A. H., Generalized Integral Transformations, Dover Publications, Inc., New York, 1987. 


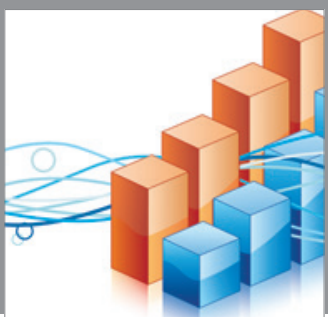

Advances in

Operations Research

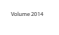

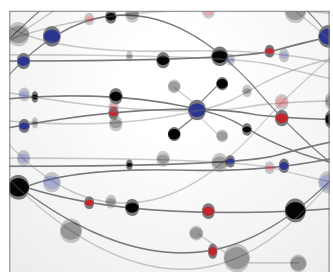

\section{The Scientific} World Journal
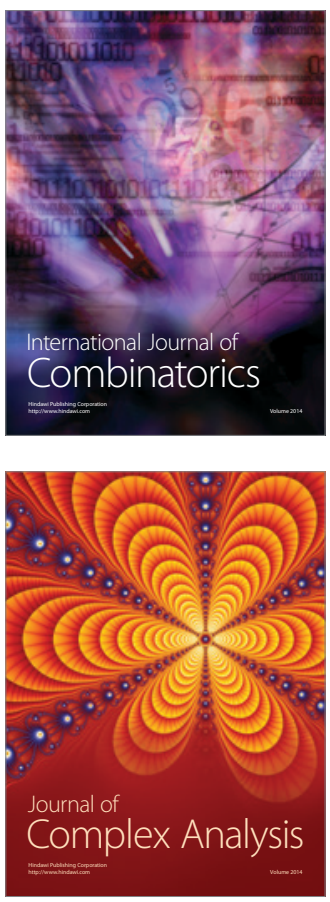

International Journal of

Mathematics and

Mathematical

Sciences
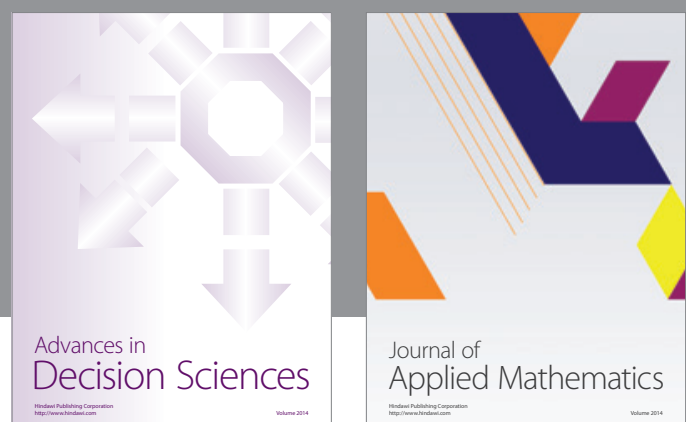

Journal of

Applied Mathematics
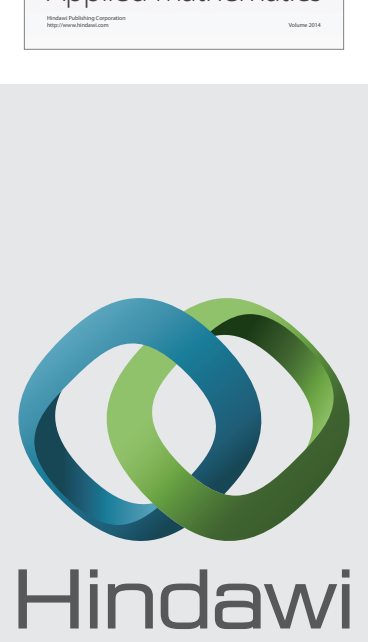

Submit your manuscripts at http://www.hindawi.com
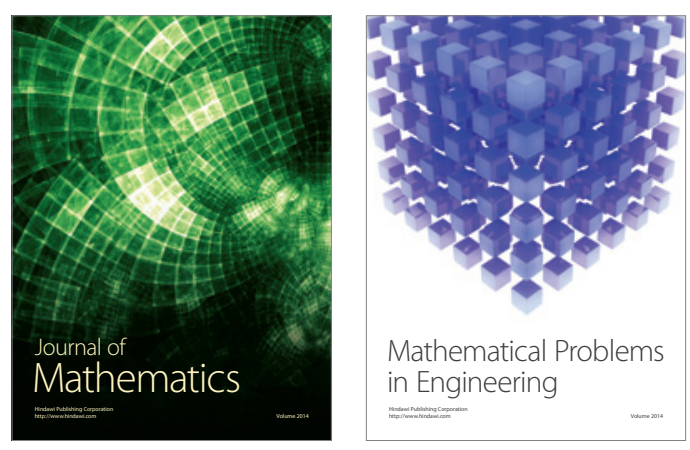

Mathematical Problems in Engineering
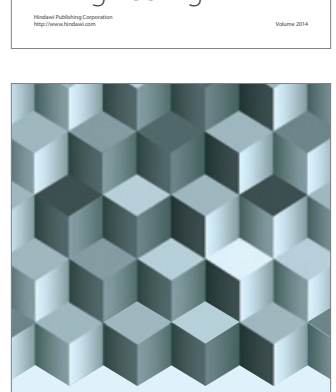

Journal of

Function Spaces
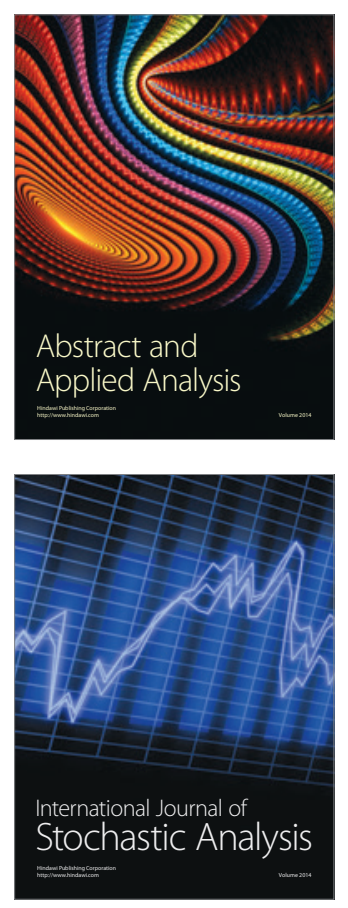

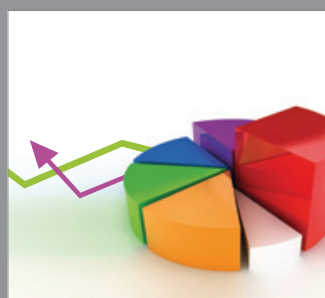

ournal of

Probability and Statistics

Promensencen
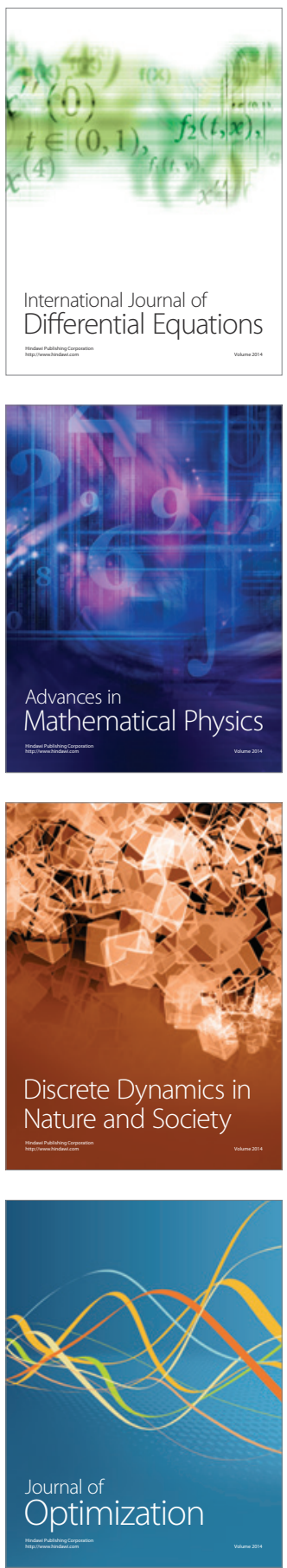